\title{
Clinical Practice Variability in Temperature Correction of Arterial Blood Gas Measurements and Outcomes in Hypothermia-Treated Patients After Cardiac Arrest
}

\author{
Samuel Waller Terman, MD, MS, ${ }^{1,2}$ Katherine S. Nicholas, BA, ${ }^{3}$ \\ Benjamin Hume, BS, ${ }^{1}$ and Robert Silbergleit, $\mathrm{MD}^{1}$
}

\begin{abstract}
Mechanical ventilation in patients treated with mild therapeutic hypothermia (MTH) for the postcardiac arrest syndrome may be challenging given changes in solubility of arterial blood gases (ABGs) with cooling. Whether ABG measurements should be temperature corrected (TC) remain unknown. We sought to describe practice variability in TC at a single institution and explored the association between TC and neurological outcome. We conducted a retrospective cohort study reviewing electronic health records of all patients treated with MTH after cardiac arrest. We examined whether the percentage of TC ABGs relative to total number of ABGs drawn for each subject during hypothermia was associated with the neurological outcome at hospital discharge and 6-12-month follow-up. The cerebral performance category of 1-2 was defined as a favorable outcome in the logistic regression models. 1223 ABGs were obtained during MTH on 122 subjects over 6 years. TC was never used in 72 subjects (59\%; no TC group), made available in 1-74\% of ABGs in 17 subjects (14\%; intermediate TC group), and made available in $\geq 75 \%$ of ABGs in 33 subjects (27\%; mostly TC group). Groups differed in the proportion of subjects with shockable presenting rhythms (47\% vs. $47 \%$ vs. $76 \%, p=0.02)$ and admitting ICU $(p=0.005)$. Favorable 6-month outcomes were more common in the mostly TC than no TC group (48\% vs. 25\%; OR [95\% CI]: 2.9 [1.2-7.1]), but not after adjustment (OR 1.5, 95\% CI 0.33-6.9). There was substantial practice variability in the temperature correction strategy. Availability of temperature-corrected ABGs was not associated with improved neurological outcomes after adjusting for covariates.
\end{abstract}

\section{Introduction}

O VER 380,000 EMERGENCY MEDICAL SERVICES- (EMS-) treated nontraumatic out-of-hospital cardiac arrests (OHCA) occur each year in the United States, with an estimated overall survival to hospital discharge with at least a moderate neurological outcome of 6.9\% (McNally et al., 2011; Roger et al., 2012). Recent data from a large randomized trial showed similar outcomes in patients cooled to two controlled subfebrile temperatures, $33^{\circ} \mathrm{C}$ versus $36^{\circ} \mathrm{C}$, following OHCA (Nielsen et al., 2013). In previous trials, mild therapeutic hypothermia (MTH) to $33^{\circ} \mathrm{C}$ was shown to benefit selected patients when compared with no temperature control (Bernard et al., 2002; Hypothermia after Cardiac Arrest Study Group, 2002; Peberdy et al., 2010; Arrich et al., 2012). Proper ventilatory support is considered important to successful postcardiac arrest management (Peberdy et al., 2010; Schneider et al., 2013), espe- cially given the potential for iatrogenic dyscarbia (Falkenbach et al., 2009) and its effect on outcomes (Schneider et al., 2013). However, there is equipoise on how this should be achieved in hypothermic patients (Hoedemaekers and van der Hoeven, 2014). As temperature decreases, the solubility of arterial blood gases (ABGs) increases, partial pressures of oxygen and carbon dioxide decrease, and $\mathrm{pH}$ rises (Alston, 2004). Ventilator management depends on how these changes are accounted for when measuring and reporting ABGs. Alternative approaches include $\mathrm{pH}$-stat in which $\mathrm{ABG}$ are temperature corrected (TC) and $\alpha$-stat in which measurements are always evaluated at $37^{\circ} \mathrm{C}$ (Alston, 2004; Bisson and Younker, 2006).

There is practice variation over whether or not to employ TC values when measuring ABGs during MTH (Bisson and Younker, 2006). The importance of these differences upon clinical outcomes in patients with relatively mild cooling after cardiac arrest has been incompletely evaluated, but

Departments of ${ }^{1}$ Emergency Medicine and ${ }^{2}$ Neurology, University of Michigan Medical School, Ann Arbor, Michigan.

${ }^{3}$ Department of Public Health Science, Medical University of South Carolina, Charleston, South Carolina.

This work was published in abstract form in Neurocritc Care 2013 19:21-S3311 and presented at the Neurocritical Care Society Annual Meeting. Philadelphia, PA, October 2013. 
remains plausible, given the sensitivity of the injured brain to $\mathrm{PaCO}_{2}$-dependent cerebral blood flow, changes in oxygen delivery and metabolism, and possibly to other electrochemical pertubations (Yundt and Diringer, 1997; Ausina et al., 1998).

The objectives of the present study are to describe practice variability in $\mathrm{TC}$ at a single institution and to use such practice variability to explore the association between TC and outcome. We hypothesize that practice variation exists even within a single institution and that such variation in TC practice will be associated with differences in outcomes.

\section{Materials and Methods}

\section{Study design}

This was an observational retrospective cohort study (Vandenbroucke et al., 2007). Subjects were classified by the percentage of $\mathrm{ABG}$ with $\mathrm{TC}$ available (i.e., $\mathrm{pH}$-stat) relative to the total number of ABGs drawn for each subject during hypothermia therapy. The main comparison constituted those with at least $75 \%$ of ABGs including $\mathrm{TC}$ values (dubbed the mostly TC group), versus those with $0 \%$ of ABGs including TC values (i.e., $100 \%$ using $\alpha$-stat, dubbed the no TC group). Those subjects with $1-74 \%$ of ABGs including TC values are dubbed the intermediate TC group.

\section{Subject selection}

Electronic health records were screened for all patients presenting to the University of Michigan Emergency Department with a presentation or diagnosis coded as OHCA, following implementation of our MTH program between August 26, 2006, and September 14, 2012. To ensure that we captured all eligible subjects, we also screened patients included on an independent $\log$ of therapeutic temperature management (TTM) equipment use. All included subjects were comatose, endotracheally intubated, and mechanically ventilated. Patients were excluded if they were younger than 18 years at the time of arrest, received temperature management therapy for a diagnosis other than cardiac arrest (i.e., rewarming after environmental hypothermia), if cooling was withdrawn immediately after implementation, or if no ABGs were available. Subjects were typically admitted to either a Cardiac Intensive Care Unit (CICU) or a Critical Care Medical Unit (CCMU) depending on the suspected etiology of the cardiac arrest.

\section{Therapeutic hypothermia}

MTH at our institution consisted of endovascular cooling (Innercool; Phillips, San Diego, CA) for 24 hours at a target of $33^{\circ} \mathrm{C}$ followed by controlled rewarming over 24 hours back to normothermia. Placement of the endovascular temperature control catheter typically occurred in the Emergency Department, but sometimes occurred in the interventional cardiology suite, or the ICU depending on the clinical situation. Cold intravenous crystalloid bolus was allowed to initiate cooling if placement of the catheter was delayed.

\section{Ventilation protocol}

Our institution executes a standard ventilation protocol managed by respiratory therapists. According to the protocol, ventilator frequency and/or tidal volumes were adjusted to maintain a $\mathrm{pH}$ of $7.30-7.45$ for most patients, except those with obstructive lung disease or ARDS may be ventilated with permissive hypercapnia to maintain a peak plateau level $<30 \mathrm{~cm} \mathrm{H} 20 . \mathrm{FiO}_{2}$ was titrated for $\mathrm{SpO}_{2}$ of $90-94 \%$.

\section{Data collection and outcomes assessment}

This project was reviewed and determined exempt by the University of Michigan Institutional Review Board. Data collection was based on Utstein recommendations (Jacobs et al., 2004) and performed according to reported standards in chart review research (Gilbert et al., 1996).

All data were abstracted from the University of Michigan electronic health records by the primary author (S.T.). The author conducting the analysis was not blinded to the study purpose or any part of the data. We reviewed and included all ABGs drawn during hypothermia therapy from the start of cooling induction to completion of rewarming. Details regarding demographics, medical history, the event and resuscitation, including shockable rhythm and time to reaching the target temperature of $33^{\circ} \mathrm{C}$, hypothermia protocol, and discharge vital status were also determined. Being classified as shockable means that an automatic external defibrillator was applied, which advised a shock, or the emergency department (ED) or emergency medical services (EMS) flowsheets/ runsheets mark the presenting arrest rhythm as either ventricular fibrillation (VF) or pulseless ventricular tachycardia (VT). Being classified as nonshockable means it was documented that an automated external defibrillator (AED) did not advise a shock, ED or EMS flowsheets/runsheets specify pulseless electrical activity (PEA) or asystole as the presenting cardiac rhythm, or else presenting rhythm and defibrillation are not specifically mentioned, but there exists sufficient medical documentation detailing resuscitative efforts such that it would be reasonable to assume that a defibrillation would have been documented if it had been delivered.

APACHE II scores were calculated on best available data. Laboratory values at admission were used to calculate APACHE II scores (Knaus et al., 1985; Ho et al., 2006). Vital signs at admission were used as well, except for temperature that was frequently missing on arrival. For consistency, the lowest temperature achieved during cooling was used.

Data were collected and managed using REDCap (Research Electronic Data Capture) electronic data capture tools hosted by the University of Michigan (Harris et al., 2009).

We determined whether ABGs included TC based on laboratory records in the electronic health record. A temperature listed for each blood gas of $37^{\circ} \mathrm{C}$ or else no temperature listed indicates that gases were run without TC. ABGs were determined to be TC for hypothermia if their laboratory documentation indicated a temperature below $37^{\circ} \mathrm{C}$. Corrected readings were obtained by applying mathematical formulas to the uncorrected reading to approximate partial pressures at a hypothermic temperature. All ABGs that have corrected readings also have uncorrected readings listed in the medical record.

Neurological outcomes were characterized by Cerebral Performance Category (CPC) (Jennett and Bond, 1975; A Randomized Clinical Study of a Calcium-Entry Blocker, 1991; Safar and Bircher, 1998). Outcomes were dichotomized as favorable (CPC 1-2, i.e., no symptoms and/or independence) or unfavorable (CPC 3-5, i.e., dependent, 
comatose, or dead) at hospital discharge and 6-12 months postdischarge. Outcomes were determined by reviewing inpatient and outpatient physician and physical/occupational therapy evaluations. Determination of CPC from chart review has been previously determined to have moderately good correlation with that determined by patient interview (Raina et al., 2008). CPCs were determined by two independent reviewers (S.T. and R.S.). Initial inter-rater agreement was $85 \%$ and $73 \%$ for nondeceased CPCs at discharge and follow-up, respectively, and when discordant, resolved by consensus. We used the first note after 6 months that provided sufficient information relevant to neurological symptoms on which to base our assessment. If no informative note existed in the relevant time interval but medical records indicated a consistent CPC before and after the interval of interest without mention of significant intervening event, such records were incorporated. If no such note existed or the subject was lost to follow-up from the University of Michigan electronic records, they were recorded as having a missing CPC. If the 6-month vital status was unavailable from medical records, we searched the Social Security Death Index Master File (accessed 5/14/2013).

\section{Statistical analysis}

Descriptive statistics were used to characterize the cohort. Continuous variables were described using medians and interquartile ranges. Categorical variables were described as absolute frequencies. To account for the fact that ABGs were drawn repeatedly over time, we have compared ABG measurements between groups using a generalized estimating equation with a repeated measures structure. Chi-squared tests and logistic regressions were used to determine associations between categories of proportion of $\mathrm{ABGs}$ that were temperature corrected and dichotomized CPC outcomes.

Our initial primary prespecified comparison was to compare those with all TC readings to those with no TC readings. However, given relatively few subjects with all pH-stat readings, our primary analysis was modified to compare $0 \%$ (no TC) versus $\geq 75 \%$ (mostly TC) available $\mathrm{pH}$-stat readings. In further analysis, we incorporated the intermediate TC group (1-74\% ABGs pH-stat) in our logistic regression in comparison to the no TC group. Covariates were included as potential confounders in our adjusted logistic regressions by being associated with the main TC category, the outcome variables, or conceptually important despite no significant association with either variable.

\section{Results}

\section{Baseline characteristics}

The population screened included 491 adult patients presenting with OHCA between 2006 and 2012 through the University of Michigan Emergency Department (Fig. 1). Of these, 134 had return of spontaneous circulation (ROSC) and were considered candidates for targeted temperature management; 123 of these had cooling initiated. Of the 123,1 had no ABGs available, so 122 met criteria and were ultimately included for analysis. Of the 122 included subjects, 118 (97\%) were known to reach a temperature of $\leq 34^{\circ} \mathrm{C}$.

Baseline characteristics are displayed in Table 1. Groups are similar, except subjects differed between the TC groups according to the proportion presenting with shockable rhythms $(p=0.02)$ and admitting ICU $(p=0.005)$.

Thirty-eight percent of the CICU subjects were categorized in the mostly TC group, as opposed to $12 \%$ of the CCMU subjects (Table 2, $p=0.003$ ). There was no difference in the number of ABGs collected per subject by the ICU category ( $p=0.29)$. Subjects in the two major ICU categories also differed by etiology of arrest $(p<0.001)$ and shockable presentation $(p<0.001)$ (Table 2$)$.

\section{$A B G$ measurements}

In total, we obtained 1223 ABGs (median [IQR]: 10 [7-13] per subject) from 122 subjects, of which 358 (29\%) included TC. The distribution in the proportion of ABGs that were TC per subject is displayed in Figure 2.

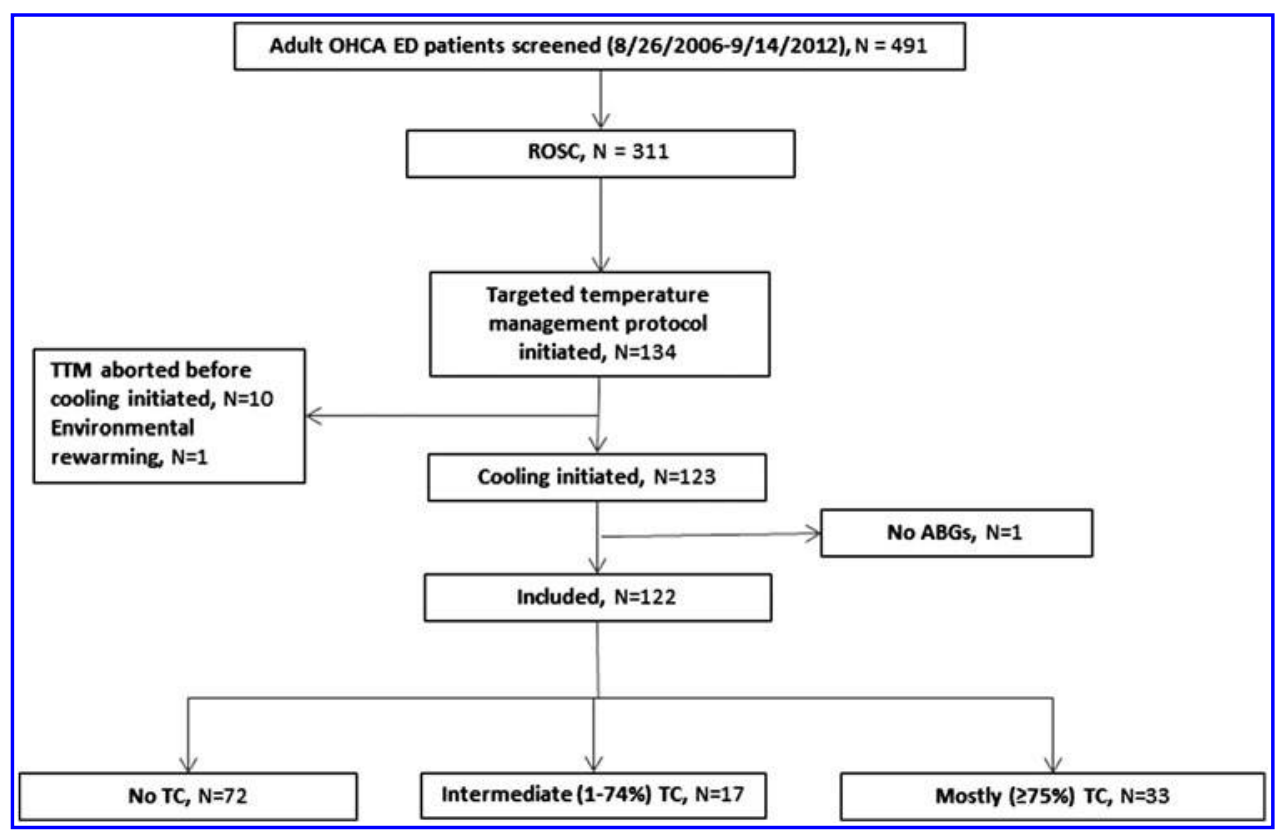

FIG. 1. Subject Selection. ABGs, arterial blood gases; ED, emergency department; OHCA, out-of-hospital cardiac arrests; ROSC, return of spontaneous circulation; TC, temperature corrected; TTM, therapeutic temperature management. 
Table 1. Baseline Characteristics

\begin{tabular}{|c|c|c|c|c|}
\hline & $\begin{array}{c}\text { No TC group } \\
(\mathrm{n}=72)\end{array}$ & $\begin{array}{l}\text { Intermediate } T C \\
\text { group }(\mathrm{n}=17)\end{array}$ & $\begin{array}{c}\text { Mostly TC } \\
\text { group }(\mathrm{n}=33)\end{array}$ & $\mathrm{p}$ \\
\hline Age, years, median (IQR) & $59(52-72)$ & $63(53-78)$ & $62(52-67)$ & 0.59 \\
\hline BMI, median (IQR) & $27(23-32)$ & $28(24-32)$ & $28(26-30)$ & 0.53 \\
\hline Sex, male, $n(\%)$ & $48(67)$ & $10(59)$ & $22(67)$ & 0.82 \\
\hline \multicolumn{5}{|l|}{$\mathrm{PMH}, n(\%)$} \\
\hline $\mathrm{MI}$ & $11(15)$ & $5(29)$ & $7(21)$ & 0.38 \\
\hline $\mathrm{CHF}$ & 12 (17) & $5(29)$ & $2(6)$ & 0.09 \\
\hline TIA or stroke ${ }^{a}$ & 7 (10) & $3(18)$ & $5(15)$ & 0.53 \\
\hline Dementia & $2(3)$ & $0(0)$ & $0(0)$ & $>0.99$ \\
\hline $\mathrm{DM}$ & $21(29)$ & $5(29)$ & $5(15)$ & 0.28 \\
\hline Dialysis & $6(8)$ & $2(12)$ & $1(3)$ & 0.37 \\
\hline \multicolumn{5}{|l|}{ Witnessed, $n(\%)$} \\
\hline Yes & $56(78)$ & $14(82)$ & $27(82)$ & $0.65^{\mathrm{b}}$ \\
\hline No & $16(22)$ & 2 (12) & $6(18)$ & \\
\hline Unknown & $0(0)$ & $1(6)$ & $0(0)$ & \\
\hline \multicolumn{5}{|l|}{ Bystander CPR, $n(\%)$} \\
\hline Yes & $51(71)$ & $9(53)$ & $25(76)$ & $0.36^{\mathrm{b}}$ \\
\hline No & $20(28)$ & $7(41)$ & $8(24)$ & \\
\hline Unknown & $1(1)$ & $1(6)$ & $0(0)$ & \\
\hline \multicolumn{5}{|l|}{ Etiology, $n(\%)$} \\
\hline $\mathrm{CV}$ & $14(19)$ & $4(24)$ & $12(36)$ & 0.42 \\
\hline Respiratory & $9(13)$ & $3(18)$ & $1(3)$ & \\
\hline Unknown & $38(53)$ & $8(47)$ & $15(45)$ & \\
\hline Other $^{\mathrm{c}}$ & $11(15)$ & $2(12)$ & $5(15)$ & \\
\hline Shockable presenting rhythm, $n(\%)^{\mathrm{d}}$ & $34(47)$ & $8(47)$ & $25(76)$ & 0.02 \\
\hline \multicolumn{5}{|l|}{$\mathrm{ICU}, n(\%)^{\mathrm{e}}$} \\
\hline CICU & $33(45)$ & $11(65)$ & $27(81)$ & 0.005 \\
\hline CCMU & $37(51)$ & $6(35)$ & $6(18)$ & \\
\hline Other & $2(3)$ & $0(0)$ & $0(0)$ & \\
\hline APACHE II, median $(\mathrm{IQR})^{\mathrm{f}}$ & $28(24-32)$ & $30(28-33)$ & $26(22-30)$ & 0.17 \\
\hline ABGs per patient, median (IQR) & $10(7-14)$ & $9(6-11)$ & $9(7-13)$ & 0.3 \\
\hline Time to target, hours, median $(\mathrm{IQR})^{\mathrm{g}}$ & $5.9(3.5-8.4)$ & $6.6(5.5-8.2)$ & $4.8(3.1-6.7)$ & 0.5 \\
\hline Withdrawal of life support, $n /$ No. of died $(\%)^{\mathrm{h}}$ & $37 / 44(84)$ & $13 / 15(87)$ & $11 / 16(73)$ & 0.69 \\
\hline
\end{tabular}

${ }^{\mathrm{a}}$ Includes ischemic or hemorrhagic stroke or TIA.

${ }^{\mathrm{b}} p$-Value excluding unknown category.

Includes intoxication ( $1 \alpha$-stat group, $3 \mathrm{pH}$-stat group), electrolyte disturbance ( $2 \alpha$-stat group), trauma ( $1 \alpha$-stat group), hanging ( $2 \alpha$-stat group), electrocution ( $2 \alpha$-stat group, $2 \mathrm{pH}$-stat group), allergic reaction $(1 \alpha$-stat group), precipitating seizure ( $\alpha \alpha$-stat group), sepsis (1 intermediate group), drowning ( 1 intermediate group), and precipitating coitus ( $1 \alpha$-stat group).

${ }^{\mathrm{d} I n c l u d e s}$ ventricular fibrillation and pulseless ventricular tachycardia. Pairwise comparisons as follows: $\alpha$-stat versus intermediate $(p>0.99), \alpha$-versus $\mathrm{pH}$-stat $(p=0.006)$, and intermediate versus $\mathrm{pH}$-stat $(p=0.04)$.

${ }^{\text {e}}$ Pairwise comparisons as follows: $\alpha$-stat versus intermediate $(p=0.46), \alpha$-versus $\mathrm{pH}$-stat $(p=0.001)$, and intermediate versus $\mathrm{pH}$-stat $(p=0.29)$.

${ }_{\mathrm{f}}^{\mathrm{f}}$ Acute Physiologic and Chronic Health Evaluation II Score.

${ }^{\mathrm{g}}$ Duration between Emergency Department arrival and consecutive recorded core body temperatures $<33.5^{\circ} \mathrm{C}$

${ }^{\mathrm{h}}$ Among those that expired.

ABGs, arterial blood gases; BMI, body mass index; CCMU, Critical Care Medical Unit; CHF, congestive heart failure; CICU, Cardiac Intensive Care Unit; CPR, cardiopulmonary resuscitation; CV, cardiovascular; DM, diabetes mellitus; IQR, interquartile range; MI, myocardial infarction; PMH, past medical history; TC, temperature corrected; TIA, transient ischemic attack.

Average ABG measurements are listed in Table 3. Carbon dioxide $\left(\mathrm{CO}_{2}\right)$ measurements were a mean $(95 \% \mathrm{CI}) 5.7$ (2.98.5) $\mathrm{mmHg}$ lower after correction, and $\mathrm{CO}_{2}$ readings differed according to the group $(p<0.0001)$. Other values did not significantly differ according to group.

\section{Neurological outcomes}

Table 4 shows outcomes by group in this cohort. No subject had a missing discharge vital status. In the no TC group, $1(1 \%)$ and $7(10 \%)$ had missing discharge and follow-up CPCs, respectively. In the mostly TC group, $0(0 \%)$ and $2(6 \%)$ had missing discharge and follow-up CPCs, respectively. No subject in the intermediate TC group had a missing CPC.

In unadjusted analysis, the intermediate TC group demonstrated lower odds of hospital survival than the other two groups (OR, 95\%: 0.21, 0.04-0.99 compared with the no TC group). Survival did not differ between the mostly TC and no TC groups. The mostly TC group had higher odds of a favorable CPC at both discharge and follow-up than the other two groups.

In Table 4, we adjusted for whether the presenting rhythm was shockable, age, sex, history of diabetes and congestive heart failure, APACHE II score, etiology, and ICU. Survival 
Table 2. Characteristics by Admitting INTENSIVE CARE UNIT

\begin{tabular}{|c|c|c|c|}
\hline & $\begin{array}{c}C I C U \\
(n=71)\end{array}$ & $\begin{array}{l}C C M U \\
(n=49)\end{array}$ & $\mathrm{p}^{\mathrm{a}}$ \\
\hline $\begin{array}{l}\text { Age, years, } \\
\text { median (IQR) }\end{array}$ & $63(54-72)$ & $58(43-72)$ & 0.11 \\
\hline BMI, median (IQR) & $27(24-30)$ & $28(24-35)$ & 0.39 \\
\hline Sex, male, $n(\%)$ & $48(68)$ & $30(61)$ & 0.47 \\
\hline \multicolumn{4}{|l|}{$\mathrm{PMH}, n(\%)$} \\
\hline MI & $17(24)$ & $6(12)$ & 0.11 \\
\hline $\mathrm{CHF}$ & $12(17)$ & 7 (14) & 0.7 \\
\hline TIA or stroke & $11(15)$ & $4(8)$ & 0.23 \\
\hline Dementia & $0(0)$ & $2(4)$ & 0.16 \\
\hline DM & $15(21)$ & $16(33)$ & 0.29 \\
\hline Dialysis & $5(7)$ & $4(8)$ & $>0.99$ \\
\hline \multicolumn{4}{|l|}{ Witnessed, $n(\%)$} \\
\hline Yes & $60(85)$ & $35(71)$ & 0.06 \\
\hline No & $10(14)$ & $14(29)$ & \\
\hline Unknown & $1(1)$ & $0(0)$ & \\
\hline \multicolumn{4}{|l|}{ Bystander CPR, $n(\%)$} \\
\hline Yes & $53(75)$ & $30(61)$ & 0.12 \\
\hline No & $17(24)$ & $18(37)$ & \\
\hline Unknown & $1(1)$ & $1(2)$ & \\
\hline \multicolumn{4}{|l|}{ Etiology, $n(\%)^{\mathrm{b}}$} \\
\hline CV & $27(38)$ & $2(4)$ & $<0.001$ \\
\hline Respiratory & $4(6)$ & 9 (18) & \\
\hline Unknown & $37(52)$ & $24(49)$ & \\
\hline Other & $3(4)$ & $14(29)$ & \\
\hline $\begin{array}{l}\text { Shockable presenting } \\
\text { rhythm }\end{array}$ & $58(82 \%)$ & $8(16)$ & $<0.001$ \\
\hline \multicolumn{4}{|l|}{ TC category, $n(\%)$} \\
\hline No TC group & $33(46)$ & $37(76)$ & 0.003 \\
\hline $\begin{array}{l}\text { Intermediate } \\
\text { TC group }\end{array}$ & $11(15)$ & $6(12)$ & \\
\hline Mostly TC group & $27(38)$ & $6(12)$ & \\
\hline $\begin{array}{l}\text { APACHE II, } \\
\text { median (IQR) }\end{array}$ & $27(22-31)$ & $29(25-32)$ & 0.06 \\
\hline $\begin{array}{l}\text { ABGs per patient, } \\
\text { median (IQR) }\end{array}$ & $10(7-13)$ & $9(6-13)$ & 0.29 \\
\hline $\begin{array}{l}\text { Time to target, } \\
\text { hours, median (IQR) }\end{array}$ & $5.0(3.3-7.9)$ & $5.9(3.8-8.4)$ & 0.11 \\
\hline $\begin{array}{l}\text { Withdrawal of life } \\
\text { support, } n / \text { No. } \\
\text { died }(\%)\end{array}$ & $26 / 33(79)$ & $34 / 40(85)$ & 0.49 \\
\hline
\end{tabular}

${ }^{a}$ Note that the other ICU group is not displayed due to a small number of subjects in that group $(n=2)$. $p$-Values compare the CICU versus CCMU populations. $p$-Values are calculated from $t$-tests for continuous variables (age, BMI, APACHE II, time to target), and either chi-square or Fisher's exact tests for categorical variables.

b Includes intoxication (4 CCMU), electrolyte disturbance (1 CCMU, 1 CICU), trauma, hanging (2 CCMU), electrocution (3 CCMU, 1 CICU), allergic reaction (1 CCMU), precipitating seizure (1 CCMU), sepsis (1 CCMU), drowning (1 CCMU), and precipitating coitus (1 CICU).

remained lower in the intermediate TC group compared with the other two groups (OR, 95\%: 0.07, 0.01-0.56 compared with the no TC group) and still did not differ between the no TC and mostly TC groups. There were no longer any significant differences between groups regarding either discharge or follow-up CPCs.

Several sensitivity analyses were performed. First, we used different comparison cutoffs. Results were similar whether we compared $0 \%(n=72)$ versus $\geq 75 \%$, as we have done here, or $<25 \%(n=74)$ versus $\geq 75 \%$. Second, we reanalyzed the data as if missing CPCs were either all favorable or

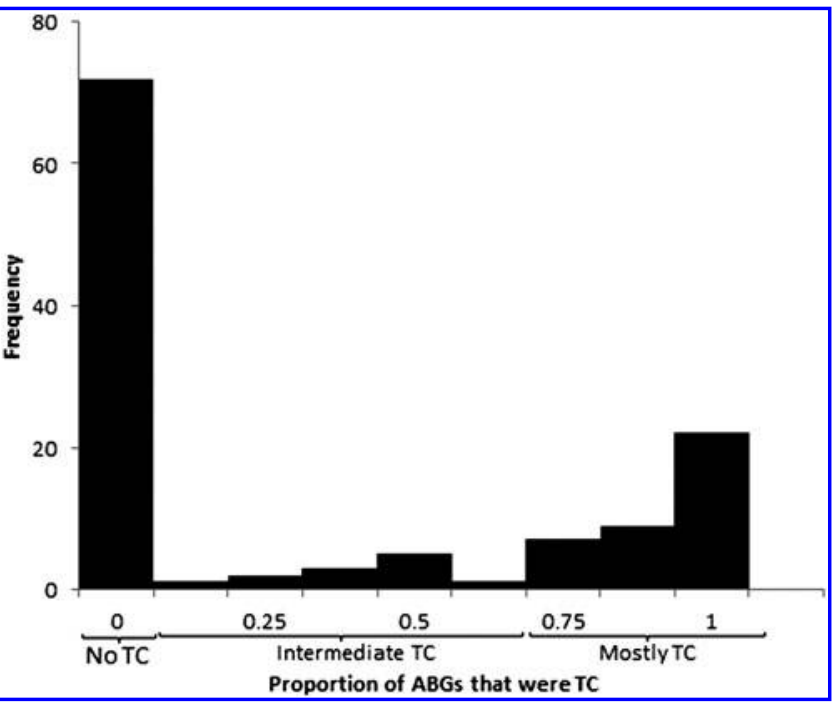

FIG. 2. Proportion of TC ABG Distribution. Brackets delineate our three study groups: the large mode at 0 represents our no TC group, in which no ABGs were TC $(n=72)$, those above 0.75 represent our mostly TC group, in which at least $75 \%$ of each subject's ABGs included TC $(n=33)$, and those in between represent the intermediate TC group, in which $1-74 \%$ of ABGs included TC $(n=17)$.

unfavorable. Results were quantitatively and qualitatively similar to the reported results. Third, we adjusted for a variety of covariate combinations. Initially, we adjusted only for presenting rhythm because it was the only factor fulfilling the statistical definition of a confounder, that is, significantly associated with both TC groups ( $p=0.02$, Tables 1 and 2$)$ and outcomes (e.g., $p<0.001$ for hospital survival). We then broadened our array of confounders to include any factor associated with either (1) the TC group [ICU $(p=0.005)]$ or (2) outcomes [for hospital survival: sex $(p=0.03)$, diabetes $(p=0.008)$, APACHE II score $(p<0.001)]$. We also then included CHF, given its borderline significant association with both the TC group $(p=0.09)$ and survival $(p=0.08)$, and also etiology, given its clinical and conceptual importance. We also ran models both including and excluding ICU, given that because neither subject in the other ICUs had a favorable CPC, including such a term that commits a quasicomplete separation of values. We also forced the TC category into the model and performed backwards model selection from the fully adjusted model with staying criteria of $p=0.05$. Ultimately, all adjusted results were both qualitatively and quantitatively similar in models where we adjusted for only presenting rhythm, the fully adjusted model, or any subset such as using backwards model selection.

\section{Discussion}

Our data confirm that substantial clinical practice variation exists even at a single institution. Our results also suggest that availability of TC ABGs was not associated with improved neurological outcomes after adjusting for covariates. Individuals with a mixture of TC methods (the intermediate TC group) demonstrated poorer hospital survival than those with predominantly TC or no TC (Table 4). It is possible that inconsistency in ventilator management, rather than which strategy is chosen, is harmful. However, caution is necessary 
Table 3. Arterial Blood Gas Measurements

\begin{tabular}{lccccc}
\hline & $\begin{array}{c}\text { Impact of corrections } \\
\text { (before-after) }^{\mathrm{a}}\end{array}$ & No TC group & Intermediate TC group & Mostly TC group & p-Value \\
\hline $\mathrm{pH}$ & $-0.05(-0.06,-0.03)$ & $7.34(7.32,7.35)$ & $7.36(7.33,7.40)$ & $7.36(7.34,7.39)$ & 0.14 \\
$\mathrm{CO}_{2}$ & $5.7(2.9,8.5)$ & $40.0(38.1,41.1)$ & $33.6(30.5,36.7)$ & $34.0(31.8,36.2)$ & $<0.0001$ \\
$\mathrm{O}_{2}$ & $17(12,21)$ & $120(112,128)$ & $111(93,129)$ & $106(94,119)$ & 0.2 \\
$\mathrm{HCO}_{3}$ & NA & $21.0(20.0,21.9)$ & $19.2(17.2,21.1)$ & $19.78(18.37,21.18)$ & 0.17 \\
\hline
\end{tabular}

Readings are displayed as mean (95\% confidence interval).

${ }^{a}$ From analysis of change score with repeated measures (uses only subjects with correction).

${ }^{\mathrm{b}}$ From main effect of group using mixed procedure with repeated measures and time as linear (uses all subjects).

when interpreting results from the intermediate TC group due to its small sample size $(n=17)$.

Arguments exist in favor of the $\mathrm{pH}$-stat (TC) method in terms of cerebral hemodynamics. $\mathrm{PaCO}_{2}$ increases $(\mathrm{pH}$ decreases) with increasing temperature (Alston, 2004). The same blood gas analyzed at a corrected temperature ( $\mathrm{pH}$-stat), therefore, appears less acidic (lower $\mathrm{PaCO}_{2}$ ) than if it were analyzed without accounting for temperature. A gas that appears less acidic would theoretically lead to less aggressive minute ventilation to achieve the same neutral targets. Total body $\mathrm{PaCO}_{2}$ and cerebral blood flow (CBF) might, therefore, be higher in a patient ventilated with the $\mathrm{pH}$-stat method (Kofstad, 1996; Kollmar et al., 2002; Pynnonen et al., 2011) by avoiding hyperventilation, which would have exerted a vasoconstrictive effect (Diringer et al., 2002; Imberti et al., 2002; Coles et al., 2007). Borrowing from literature performed in other than populations, there are possibly improved outcomes with $\mathrm{pH}$-stat in hypothermic cardiopulmonary bypass patients (du Plessis et al., 1997; Laussen et al., 2002) and increased tissue oxygenation with $\mathrm{pH}$-stat in animal studies (Duebener et al., 2002; Markowitz et al., 2007; Pirzadeh et al., 2011). Animal (Kollmar et al., 2002) and human (Kollmar et al., 2009) studies in ischemic stroke have demonstrated this phenomenon of reduced cerebral infarct volume and higher $\mathrm{CBF}$ when ventilated using $\mathrm{pH}$ - versus $\alpha$-stat methods.

Arguments also exist in favor of the $\alpha$-stat method. Proponents of the $\alpha$-stat method suggest that $\mathrm{pH}$-stat may increase $\mathrm{CBF}$ beyond actual metabolic requirements, which may disrupt autoregulation, increase cerebral microembolization, and pro- mote intracranial hypertension (Kofstad, 1996). Furthermore, the $\alpha$-stat method theoretically maintains electrochemical neutrality (i.e., constant $\left[\mathrm{H}^{+}\right]:\left[\mathrm{OH}^{-}\right]$) during hypothermia important for cellular functions (Laussen et al., 2002). Other data suggest less cognitive dysfunction following cardiopulmonary bypass operations using the $\alpha$-stat than $\mathrm{pH}$-stat (Murkin et al., 1995). Finally, temperature correction may add an unnecessary layer of complexity if there is no clinical advantage to $\mathrm{pH}$-stat management.

The clinical effect of $\mathrm{pH}$-stat versus $\alpha$-stat in OHCA patients with ROSC treated with hypothermia has not been adequately reported. Voicu et al. (2014) reported differences in jugular vein blood saturation, cerebral oxygen extraction, and CBF between groups, but did not compare clinical outcomes between TC strategies, given their crossover study design. Eastwood et al. (2015) reported that the TC method influences blood gas readings, unsurprising since a mathematical formula converts the two methods. They generally found no significant differences in outcomes according to whether patients treated with $\alpha$-stat management would have been classified differently had the $\mathrm{pH}$-stat method been used. Their methods state that they use $\alpha$-stat (but not pH-stat) clinically; therefore, their study does not actually explore the effect of practice variability in clinical care between these two methods as we have done, and $26 \%$ of their subjects were not actually cooled.

The relevance of temperature correction may change in the future, given recent data from the TTM trial suggesting that a targeted temperature management at $36^{\circ} \mathrm{C}$ conveys the same

Table 4. Neurological Outcomes

\begin{tabular}{|c|c|c|c|c|}
\hline & $\mathrm{n}^{\mathrm{a}}$ & $\begin{array}{c}\text { Favorable } \\
\text { outcome, } \mathrm{n}(\%)\end{array}$ & $\begin{array}{l}\text { Unadjusted, } \\
\text { OR }(95 \% \text { CI })\end{array}$ & $\begin{array}{c}\text { Adjusted, } \\
\text { OR }(95 \% C I)^{\mathrm{b}}\end{array}$ \\
\hline \multicolumn{5}{|l|}{ Hospital survival } \\
\hline No TC group & 72 & $28(39)$ & 1 (Ref) & 1 (Ref) \\
\hline Intermediate TC group & 17 & $2(12)$ & $0.21(0.04-0.99)$ & $0.07(0.01-0.56)$ \\
\hline Mostly TC group & 33 & $18(55)$ & $1.9(0.82-4.3)$ & $0.53(0.15-1.9)$ \\
\hline \multicolumn{5}{|l|}{ Discharge CPC } \\
\hline No TC group & 71 & $15(21)$ & 1 (Ref) & 1 (Ref) \\
\hline Intermediate TC group & 17 & $2(12)$ & $0.50(0.10-2.4)$ & $0.51(0.06-4.5)$ \\
\hline Mostly TC group & 33 & $14(42)$ & $2.8(1.1-6.7)$ & $2.1(0.52-8.8)$ \\
\hline \multicolumn{5}{|l|}{ Follow-up CPC } \\
\hline No TC group & 65 & $16(25)$ & 1 (Ref) & 1 (Ref) \\
\hline Intermediate TC group & 17 & $2(12)$ & $0.41(0.08-2.0)$ & $0.20(0.02-2.1)$ \\
\hline Mostly TC group & 31 & $15(48)$ & $2.9(1.2-7.1)$ & $1.5(0.33-6.9)$ \\
\hline
\end{tabular}

${ }^{a} n$ not always 72 and 33 for $\alpha$ - and $\mathrm{pH}$-stat groups due to missing values.

${ }^{\mathrm{b}}$ Adjusted for whether presenting rhythm was shockable, age, sex, history of diabetes, history of CHF, APACHE II score, etiology, and ICU. CI, confidence interval; CPC, cerebral performance category; ICU, intensive care unit; OR, odds ratio. 
level of neuroprotection as a target of $33^{\circ} \mathrm{C}$ (Nielsen et al., 2013).

Our study has a number of limitations. We do not have available exactly what ventilator changes were made in response to each blood gas for all patients. However, our institution does have a standard ventilator protocol whereby respiratory rate and/or tidal volumes were adjusted to achieve a normal $\mathrm{pH}$ using either the uncorrected values when no corrected values were available or the corrected values when corrected values were available. The fact that TC has a statistically significant change on $\mathrm{pH}$ and yet $\mathrm{pH}$ is similar across groups (Table 3) supports that clinicians reacted appropriately at the bedside to achieve the same targeted acid/ base values across groups based on the available gases. Presumably, similar $\mathrm{pH}$ readings in the no $\mathrm{TC}$ versus the mostly TC groups actually reflect that the mostly TC was in reality slightly more acidic than the no TC group, since TC makes a gas appear more basic. Therefore, in theory, there was in fact a physiological difference between groups that we may use to test whether such a physiological difference was correlated with outcomes, although this relationship is complex. Another limitation is the retrospective nature of outcome measures and the limited numbers of patients in this study leading to a high risk of bias. CPCs were based on the electronic medical records review, which although a common practice in cardiac arrest literature, may fall short compared with a prospective more detailed neurological assessment.

Our study has a number of strengths. Our study design and primary outcomes build upon previous reports, which used animal models, physiological surrogate patient endpoints, and retrospective group classification. We have done so by using practice variability to assess differences in multiple clinically meaningful patient outcomes. In addition, we included a range of etiologies (both cardiac and noncardiac), rhythms (shockable and nonshockable), and ICUs to explore a wider range of practice space, which increases the generalizability of our study.

\section{Conclusion}

There was substantial practice variability in the temperature correction strategy. Availability of TC ABGs was not associated with improved neurological outcomes after adjusting for covariates.

\section{Acknowledgments}

The authors thank Dr. Brahmajee K. Nallamothu, Jennifer Dammeyer, and Dr. Kyle Gunnerson for their article suggestions. They thank the Internal Medicine Pulmonary Blood Gas Laboratory for the help in data collection and medical records interpretation. The authors also thank Dr. Valerie L. Durkalski-Mauldin for her statistical review of this article.

\section{Authors' Contributions}

S.W.T. contributed to literature search, data collection, study design, analysis of data, article preparation, and review of the article. K.S.N. contributed to analysis of data and review of the article. B.H. contributed to data collection. R.S. contributed to literature search, study design, article preparation, and review of the article.

\section{Author Disclosure Statement}

No competing financial interests exist.

\section{References}

Alston TA. Blood gases and $\mathrm{pH}$ during hypothermia: the "-stats." Int Anesthesiol Clin 2004;42:73-80.

Arrich J, Holzer M, Havel C, Mullner M, Herkner H. Hypothermia for neuroprotection in adults after cardiopulmonary resuscitation. Cochrane Database Syst Rev 2012;9: CD004128.

Ausina A, Baguena M, Nadal M, et al. Cerebral hemodynamic changes during sustained hypocapnia in severe head injury: can hyperventilation cause cerebral ischemia? Acta Neurochir Suppl 1998;71:1-4.

Bernard SA, Gray TW, Buist MD, et al. Treatment of comatose survivors of out-of-hospital cardiac arrest with induced hypothermia. N Engl J Med 2002;346:557-563.

Bisson J, Younker J. Correcting arterial blood gases for temperature: (when) is it clinically significant? Nurs Crit Care 2006;11:232-238.

Coles JP, Fryer TD, Coleman MR, et al. Hyperventilation following head injury: effect on ischemic burden and cerebral oxidative metabolism. Crit Care Med 2007;35:568-578.

Diringer MN, Videen TO, Yundt K, et al. Regional cerebrovascular and metabolic effects of hyperventilation after severe traumatic brain injury. J Neurosurg 2002;96:103-108.

du Plessis AJ, Jonas RA, Wypij D, et al. Perioperative effects of alpha-stat versus $\mathrm{pH}$-stat strategies for deep hypothermic cardiopulmonary bypass in infants. J Thorac Cardiovasc Surg 1997;114:991-1000; discussion 1000-1001.

Duebener LF, Hagino I, Sakamoto T, et al. Effects of $\mathrm{pH}$ management during deep hypothermic bypass on cerebral microcirculation: alpha-stat versus pH-stat. Circulation 2002;106 (12 Suppl 1):I103-I108.

Eastwood GM, Suzuki S, Lluch C, Schneider AG, Bellomo R. A pilot assessment of alpha-stat vs $\mathrm{pH}$-stat arterial blood gas analysis after cardiac arrest. J Crit Care 2015;30:138-144.

Falkenbach P, Kamarainen A, Makela A, et al. Incidence of iatrogenic dyscarbia during mild therapeutic hypothermia after successful resuscitation from out-of-hospital cardiac arrest. Resuscitation 2009;80:990-993.

Gilbert EH, Lowenstein SR, Koziol-McLain J, Barta DC, Steiner J. Chart reviews in emergency medicine research: Where are the methods? Ann Emerg Med 1996;27:305-308.

Harris PA, Taylor R, Thielke R, Payne J, Gonzalez N, Conde JG. Research electronic data capture (REDCap) — a metadatadriven methodology and workflow process for providing translational research informatics support. J Biomed Inform 2009;42:377-381.

Ho KM, Dobb GJ, Knuiman M, Finn J, Lee KY, Webb SA. A comparison of admission and worst 24-hour Acute Physiology and Chronic Health Evaluation II scores in predicting hospital mortality: a retrospective cohort study. Crit Care 2006; $10:$ R4.

Hoedemaekers C, van der Hoeven JG. Is alpha-stat or pH-stat the best strategy during hypothermia after cardiac arrest?*. Crit Care Med 2014;42:1950-1951.

Hypothermia after Cardiac Arrest Study Group. Mild therapeutic hypothermia to improve the neurologic outcome after cardiac arrest. N Engl J Med 2002;346:549-556.

Imberti R, Bellinzona G, Langer M. Cerebral tissue PO2 and $\mathrm{SjvO} 2$ changes during moderate hyperventilation in patients 
with severe traumatic brain injury. J Neurosurg 2002;96: 97-102.

Jacobs I, Nadkarni V, Bahr J, et al. Cardiac arrest and cardiopulmonary resuscitation outcome reports: update and simplification of the Utstein templates for resuscitation registries. A statement for healthcare professionals from a task force of the International Liaison Committee on Resuscitation. Resuscitation 2004;63:233-249.

Jennett B, Bond M. Assessment of outcome after severe brain damage. Lancet 1975;1:480-484.

Knaus WA, Draper EA, Wagner DP, Zimmerman JE. APACHE II: a severity of disease classification system. $\underline{\text { Crit Care Med }}$ 1985;13:818-829.

Kofstad J. Blood gases and hypothermia: some theoretical and practical considerations. Scand J Clin Lab Invest Suppl 1996; 224:21-26.

Kollmar R, Frietsch T, Georgiadis D, et al. Early effects of acid-base management during hypothermia on cerebral infarct volume, edema, and cerebral blood flow in acute focal cerebral ischemia in rats. Anesthesiology 2002;97:868-874.

Kollmar R, Georgiadis D, Schwab S. Alpha-stat versus pH-stat guided ventilation in patients with large ischemic stroke treated by hypothermia. Neurocrit Care 2009;10:173-180.

Laussen PC. Optimal blood gas management during deep hypothermic paediatric cardiac surgery: alpha-stat is easy, but $\mathrm{pH}$ stat may be preferable. Paediatr Anaesth 2002;12:199-204.

Markowitz SD, Mendoza-Paredes A, Liu H, et al. Response of brain oxygenation and metabolism to deep hypothermic circulatory arrest in newborn piglets: comparison of $\mathrm{pH}$-stat and alpha-stat strategies. Ann Thorac Surg 2007;84:170-176.

McNally B, Robb R, Mehta M, Vellano K, Valderrama AL, Yoon PW, Sassoon C, Crouch A, Perez AB, Merritt R, Kellermann A; Centers for Disease Control and Prevention. Out-of-hospital cardiac arrest surveillance-cardiac arrest registry to enhance survival (CARES), United States, October 1, 2005-December 31, 2010. MMWR Surveill Summ 2011; 60:1-19.

Murkin JM, Martzke JS, Buchan AM, Bentley C, Wong CJ. A randomized study of the influence of perfusion technique and pH management strategy in 316 patients undergoing coronary artery bypass surgery. II. Neurologic and cognitive outcomes. J Thorac Cardiovasc Surg 1995;110:349-362.

Nielsen N, Wetterslev J, Cronberg T, et al. Targeted temperature management at 33 degrees $\mathrm{C}$ versus 36 degrees $\mathrm{C}$ after cardiac arrest. N Engl J Med 2013;369:2197-2206.

Peberdy MA, Callaway CW, Neumar RW, et al. Part 9: post-cardiac arrest care: 2010 American Heart Association Guidelines for Cardiopulmonary Resuscitation and Emergency Cardiovascular Care. Circulation 2010;122(18 Suppl 3):S768-S786.

Pirzadeh A, Schears G, Pastuszko P, et al. Effect of deep hypothermic circulatory arrest followed by low-flow cardio- pulmonary bypass on brain metabolism in newborn piglets: comparison of pH-stat and alpha-stat management. Pediatr Crit Care Med 2011;12:e79-e86.

Pynnonen L, Falkenbach P, Kamarainen A, Lonnrot K, YliHankala A, Tenhunen J. Therapeutic hypothermia after cardiac arrest - cerebral perfusion and metabolism during upper and lower threshold normocapnia. Resuscitation 2011;82: 1174-1179.

Raina KD, Callaway C, Rittenberger JC, Holm MB. Neurological and functional status following cardiac arrest: method and tool utility. Resuscitation 2008;79:249-256.

A randomized clinical study of a calcium-entry blocker (lidoflazine) in the treatment of comatose survivors of cardiac arrest. Brain Resuscitation Clinical Trial II Study Group. N Engl J Med 1991;324:1225-1231.

Roger VL, Go AS, Lloyd-Jones DM, et al. Heart disease and stroke statistics-2012 update: a report from the American Heart Association. Circulation 2012;125:e2-e220.

Safar P, Bircher NG. Cardiopulmonary Cerbral Resuscitation: Basic and Advanced Cardiac and Trauma Life Support: An Introduction to Resuscitation Medicine. Vol 267. 3rd ed. London: Saunders, 1998.

Schneider AG, Eastwood GM, Bellomo R, et al. Arterial carbon dioxide tension and outcome in patients admitted to the intensive care unit after cardiac arrest. Resuscitation 2013;84: 927-934.

Vandenbroucke JP, von Elm E, Altman DG, et al. Strengthening the reporting of observational studies in epidemiology (STROBE): explanation and elaboration. PLoS Med 2007; 4:e297.

Voicu S, Deye N, Malissin I, et al. Influence of alpha-stat and $\mathrm{pH}$-stat blood gas management strategies on cerebral blood flow and oxygenation in patients treated with therapeutic hypothermia after out-of-hospital cardiac arrest: a crossover study. Crit Care Med 2014;42:1849-1861.

Yundt $\mathrm{KD}$, Diringer $\mathrm{MN}$. The use of hyperventilation and its impact on cerebral ischemia in the treatment of traumatic brain injury. Crit Care Clin 1997;13:163-184.

Address correspondence to: Robert Silbergleit, $M D$ Department of Emergency Medicine University of Michigan Medical School Neuro Emergencies Research 24 Frank Lloyd Wright Drive Lobby H, Suite 3100, Box 381 Ann Arbor, MI 48105

E-mail: robert.silbergleit@umich.edu 
This article has been cited by:

1. Pieter F. Fouche, Jestin N. Carlson, Arindam Ghosh, Kristina M. Zverinova, Suhail A. Doi, Jon C. Rittenberger. 2017. Frequency of adjustment with comorbidity and illness severity scores and indices in cardiac arrest research. Resuscitation 110, 56-73. [CrossRef] 